\title{
SOME CONTINUITY PROPERTIES OF LINEAR TRANSFORMATIONS IN NORMED SPACES
}

\author{
by R. W. CROSS
}

(Received 10 February, 1987)

Let $X$ and $Y$ be normed spaces and let $L(X, Y)$ denote the set of linear transformations (henceforth called "operators") $T$ with domain a linear subspace $D(T)$ of $X$ and range $R(T)$ contained in $Y$. The restriction of $T$ to a subspace $E$ is denoted by $T \mid E$; by the usual convention $T|E=T| E \cap D(T)$. For a given linear subspace $E$ the family of infinite dimensional subspaces of $E$ is denoted by $\mathscr{I}(E)$. An operator $T$ is said to have a certain property $\mathbb{P}$ ubiquitously if every $E \in \mathscr{I}(X)$ contains an $F \in \mathscr{T}(E)$ for which $T \mid F$ has property $\mathbb{P}$. For example, $T$ is ubiquitously continuous if each $E \in \mathscr{I}(X)$ contains an $F \in \mathscr{I}(E)$ for which $T \mid F$ is continuous. In the present note we shall characterize ubiquitous continuity, isomorphy, precompactness and smallness. A subspace of $X$ is called a principal subspace if it is closed and of finite codimension in $X$. The restriction of an operator to a principal subspace will be called a principal restriction. The symbol $T$ will always denote an arbitrary operator in $L(X, Y)$.

LEMMA 1. If $M$ and $E$ are subspaces of $X$ and if $\operatorname{codim} E<\infty$, then $M=M \cap E \oplus F$ for some finite dimensional subspace $F$. injective.

Proof. The map of $M / M \cap E$ into $X / E$ given by $m+M \cap E \rightarrow m+E(m \in M)$ is

A restriction $T \mid M$ of $T$ is said to be nontrivial if $M \cap D(T)$ is infinite dimensional.

LEMMA 2. The operator $T$ has a principal restriction having a continuous inverse if and only if $T$ has no nontrivial precompact restriction.

Proof. The "if" part is contained in the Kato-Goldberg result [3, p. 80]. For the converse, suppose that $M$ is a principal subspace for which $T \mid M$ has a continuous inverse and let $E$ be a subspace such that $T \mid E$ is precompact. Then $T \mid M \cap E$ is an isomorphism and hence $M \cap E \cap D(T)$ is finite dimensional. Therefore $E \cap D(T)$ is finite dimensional by Lemma 1 .

Corollary 3. (See [2].) Any two norms defined on an infinite dimensional linear space are comparable on some infinite dimensional subspace.

Proof. Consider the appropriate identity map.

With a given operator $T$ we associate the graph operator $G$ of $T$ as follows. Let $X_{T}$ be the linear space $D(T)$ normed by $\|x\|_{T}=\|x\|+\|T x\|$ and define the operator $G: X_{T} \rightarrow X$ by $G x=x\left(x \in X_{T}\right)$. Observe that $T$ is continuous if and only if $G$ is an isomorphism.

Glasgow Math. J. 30 (1988) 243-247. 
THEOREM 4. The operator $T$ is ubiquitously continuous if and only if $T$ is continuous on some subspace of finite codimension.

Proof. We may clearly suppose $\operatorname{dim} D(T)=\infty$. Suppose $T$ is ubiquitously continuous. Let $E \in \mathscr{I}(D(T))$. There exists $F \in \mathscr{I}(E)$ making $T \mid F$ continuous. Then $G^{-1} \mid F$ is an isomorphism. Consequently $G$ has no nontrivial precompact restriction. Hence by Lemma 2 a principal subspace $M$ of $X_{T}$ exists for which $G \mid M$ is an isomorhism. Then $T \mid G M$ is continuous. If $N$ is a subspace of $X$ complementary to $D(T)$ then $G M \oplus N$ is a finite codimensional subspace upon which $T$ is continuous.

Conversely let $T \mid E$ be continuous where $\operatorname{codim} E<\infty$ and let $M \in \mathscr{I}(X)$. Then $E \cap M \in \mathscr{I}(X)$ by Lemma 1 , and $T \mid E \cap M$ is continuous. Thus $T$ is ubiquitously continuous.

THEOREM 5. The following statements are equivalent.

(i) $T$ is ubiquitously an isomorphism.

(ii) $T$ is an isomorphism on some subspace of finite codimension.

(iii) $T$ is continuous on some subspace of finite codimension and $T$ has no nontrivial precompact restriction.

Proof. The implication (i) $\Rightarrow$ (iii) is immediate from Theorem 4. Assume (iii). Then by Lemma 2 there exists a finite codimensional subspace $M$ of $X$ for which $T \mid M$ has a continuous inverse. If $E$ is a finite codimensional subspace making $T \mid E$ continuous, then $\operatorname{codim}(E \cap M)<\infty$ (Lemma 1$)$ and $T \mid E \cap M$ is an isomorphism. Hence (iii) $\Rightarrow$ (ii). The proof that (ii) $\Rightarrow$ (i) is similar to the corresponding part of the proof of Theorem 4 .

Proposition 6. Let $Z$ be a subspace of $X$. For each principal subspace $M$ of $Z$ there exists a principal subspace $M_{0}$ of $X$ such that $M=M_{0} \cap Z$.

Proof. Let $M$ be a principal subspace of $Z$. There exists a finite dimensional subspace $F$ of $Z$ such that $M \oplus F=Z$. Let $x_{1}, \ldots, x_{n}$ be a basis for $F$. Choose $f_{1}, \ldots, f_{n} \in X^{\prime}$ such that $f_{i}\left(x_{j}\right)=\delta_{i j}$ and $f_{i}(m)=0$ for $m \in M(i, j \leq n)$; this is possible since $F \cap \vec{M}=(0)$. Then $M_{0}=\bigcap_{i \leq m} f_{i}^{-1}(0)$ is a principal subspace of $X$ with $M=M_{0} \cap Z$.

LEMMA 7. If $X=M \oplus N$, where $M$ is a principal subspace, then the projection of $X$ onto $M$ with null space $N$ is bounded.

Proof. Let $\left\{x_{1}, \ldots, x_{n}\right\}$ be a basis for $N$ and let $N_{i}=\operatorname{sp}\left\{x_{1}, \ldots, x_{i-1}\right.$, $\left.x_{i+1}, \ldots, x_{n}\right\}$. Since $M+N_{i}$ is closed for each $i$ (see e.g. [3, p. 16]) there exists by the Hahn-Banach theorem an $f_{i} \in X^{\prime}$ such that $f_{i}\left(x_{i}\right)=1$ and $f_{i}(x)=0$ for $x \in M+N_{i}$. Define $Q=\sum_{i \leq n} f_{i} \otimes x_{i}$. Then $Q$ is a bounded projection with range $N$ and null space $M$, and $P=I-Q$ is the required projection.

COROllary 8. If there exists a principal subspace $M$ for which $T \mid M$ is continuous, then $T$ is continuous. 
A characterisation of bounded semi-Fredholm operators ( $\phi_{+}$-operators) between Banach spaces will now be given. An operator $T$ is called a $\phi_{+}$-operator if its null space $N(T)$ is finite dimensional and $R(T)$ is closed.

Theorem 9. Let $X$ and $Y$ be Banach spaces and let $T \in L(X, Y)$ be an everywhere defined (injective) operator. The following statements are equivalent.

(i) $T$ is a bounded $\phi_{+}$-operator (resp., an isomorphism).

(ii) $T$ is bounded and ubiquitously an isomorphism.

(iii) $T$ has a principal restriction which is an isomorphism.

Proof. Assume (ii). Then by Lemma 2 there exists a principal subspace $M$ making $T \mid M$ an isomorphism. Hence (ii) $\Rightarrow$ (iii).

Assume (iii). The implication (iii) $\Rightarrow$ (i) in the case when $T$ is bounded is a well known classical result (cf. [1]). Hence, by Corollary 8 , (iii) $\Rightarrow$ (i).

Assume (i). Then there exists a principal subspace $M$ such that $X=M \oplus N(T)$. By the Closed Graph Theorem, $T \mid M$ is an isomorphism. Hence (i) $\Rightarrow$ (ii).

THEOREM 10. Let $T$ be injective and everywhere defined on a Banach space. If $T$ is bounded, and if $T^{-1}$ is continuous on a subspace of finite codimension, then $T$ is an isomorphism.

Proof. Let $T$ be bounded and let $E$ be a finite codimensional subspace of $Y$ such that $T^{-1} \mid E$ is continuous. Then $T^{-1} E$ has finite codimension in $X$, and $T \mid T^{-1} E$ has a continuous inverse. Lemma 1 now implies that $T$ has no nontrivial precompact restriction. Hence, by Lemma 2, there is a principal subspace $M$ of $X$ for which $T \mid M$ is an isomorphism. But $M$ is complete. Therefore $T M$ is complete and hence is a principal subspace of $R(T)$ for which $T^{-1} \mid T M$ is continuous. Therefore $T^{-1}$ is continuous by Corollary 8 .

To show that completeness is essential in Theorem 10 we give an example of a bounded everywhere defined operator which is not an isomorphism, yet has a principal restriction which is an isomorphism. Let $f$ be a discontinuous linear functional with domain $X$, and let $G: X_{f} \rightarrow X$ be the graph operator associated with $f$. Then $G^{-1}$ is unbounded. However, $G^{-1} \mid N(f)$ is an isometry. Since $\operatorname{codim} N(f)=1$, it follows from Lemma 2 and Theorem 5 that $G$ has a principal restriction which is an isomorphism.

COROllary 11. Let $X_{T}$ be complete. If $T$ is ubiquitously continuous then $T$ is continuous.

Proof. If $T$ is ubiquitously continuous, then $G^{-1}$ is continuous on some subspace of finite codimension by Theorem 4 . Hence, by Theorem 10, $G$ is an isomorphism, or, equivalently, $T$ is continuous.

We remark that Corollary 11 fails without the completeness assumption; for example, every discontinuous linear functional is ubiquitously continuous. 
If $X$ and $Y$ are complete, then $X_{T}$ is complete if and only if $T$ is closed. Hence we have the following corollary.

Corollary 12. Let $X$ and $Y$ be complete. If $T$ is closed and ubiquitously continuous, then $T$ is continuous.

Assuming that $X$ and $Y$ are Banach spaces and that $T$ is everywhere defined and injective, L. Drewnowski [1] asks whether $T$ is an isomorphism whenever it has the property that for each closed subspace $E \in \mathscr{I}(X)$ there exists $F \in \mathscr{I}(E)$ for which $T \mid \bar{F}$ is an isomorphism. The following example shows that if "closed subspace $E$ " is replaced by "principal subspace $E$ " in the above, then $T$ need not be bounded.

EXAMPLE 13. There exists an unbounded everywhere defined injective and surjective operator $T: l_{2} \rightarrow l_{2}$ such that every principal subspace of $l_{2}$ contains an infinite dimensional closed subspace $F$ such that $T \mid F$ is an isomorphism.

Let $M$ and $N_{1}$ be a pair of closed mutually orthogonal infinite dimensional subspaces of $l_{2}$ such that $M+N_{1}=l_{2}$ (where $\dot{+}$ denotes the orthogonal sum), and select a dense proper subspace $N$ of $N_{1}$ so that $N_{1}=K+N$ where $K$ is one-dimensional ( $N$ will be the null space of a discontinuous linear functional on $\left.N_{1}\right)$. On $l_{2}=M+(K+N)$ define $P$ to be the projection of $l_{2}$ onto $M+K$ with null space $N$. $P$ is unbounded since its null space $N(P)$ is not closed. Let $T=I+P$. Then $T$ is unbounded. Also, $T \mid(M+N)$ is an isomorphism; indeed for $m \in M, n \in N$ we have $\|m+n\|^{2}=\|m\|^{2}+\|n\|^{2} \leq\|2 m\|^{2}+$ $\|n\|^{2}=\|2 m+n\|^{2}=\|T(m+n)\|^{2} \leq 4\|m+n\|^{2}$ so $\|m+n\| \leq\|T(m+n)\| \leq 2\|m+n\|$. Now let $E$ be a principal subspace. Then $M=M \cap E \oplus W$ where $\operatorname{dim} W<\infty$ by Lemma 1 . Hence $F=M \cap E$ is infinite dimensional and has the required property.

We shall now characterize ubiquitously precompact operators. Such an operator will be continuous on a finite codimensional subspace by Theorem 3 . An operator $T$ will be called strictly singular if there is no infinite dimensional subspace $M$ of $D(T)$ for which $T \mid M$ has a continuous inverse; this is a generalisation of the classical definition (see [4]). Any discontinuous linear functional is an example of an unbounded strictly singular operator. We shall call $T$ ubiquitously small if for each $\varepsilon>0$ and each $E \in \mathscr{G}(X)$ there exists $F \in \mathscr{I}(E)$ such that $\|T \mid F\|<\varepsilon$ unless $F \cap D(T)$ is finite dimensional. The theorem below is a generalisation of III.2.1 of [3].

THEOREM 14. The following statements are equivalent.

(i) $T$ is ubiquitously precompact,

(ii) $T$ is ubiquitously small.

(iii) $T$ is ubiquitously strictly singular.

(iv) $T$ is strictly singular.

Proof. Assume (i). By Theorem 3 there exists a finite codimensional subspace $E$ for which $T \mid E$ is continuous. Since $T \mid E$ is ubiquitously precompact, there exists $F \in \mathscr{I}(E)$ such that $T \mid F$ is precompact. In particular, $T \mid F$ is continuous and precompact on its domain $F \cap D(T)$, and hence ubiquitously small on $F \cap D(T)$ by ([3], loc. cit.). This shows that $T$ is ubiquitously small. Thus (i) $\Rightarrow$ (ii) by Lemma 1 . Next assume $T$ is not 
strictly singular and let $M \in \mathscr{I}(D(T))$ be such that $T \mid M$ has a continuous inverse. Then $\|T m\| \geq c\|m\|$ for some $c>0$ and all $m \in M$. Therefore $T$ is not ubiquitously small. Hence (ii) $\Rightarrow$ (iv). Next, assume $T$ is not ubiquitously precompact. Then there exists an infinite dimensional subspace $M$ such that $T \mid M$ has no precompact restriction to any infinite dimensional subspace of $M$. Lemma 2 now implies that $T \mid M \cap N$ has a continuous inverse for some principal subspace $N$ of $M$. Since evidently $\operatorname{dim}(M \cap D(T))=\infty$, it follows from Lemma 1 that $T \mid M$ (and hence $T$ ) is not strictly singular. Therefore (iv) $\Rightarrow$ (i). Finally, the equivalence of (i) and (iv) implies immediately the equivalence of (iii) and (iv).

Theorem 14 implies in particular that the sum of two strictly singular operators is strictly singular, and that every strictly singular operator is continuous on some subspace of finite codimension.

\section{REFERENCES}

1. L. Drewnowski, Some characterisations of semi-Fredholm operators, Comment. Math. Prace. Mat. 24 (1984), 215-218.

2. L. Drewnowski, Any two norms are somewhere comparable, Funct. Approx. Comment. Math. 7 (1979), 13-14.

3. S. Goldberg, Unbounded linear operators, (McGraw-Hill, 1966).

4. T. Kato, Perturbation theory for nullity, deficiency and other quantities of linear operators, J. Analyse Math. 6 (1958), 261-322.

UNIVERSITY OF CAPE TOWN

RONDEBOSCH

SOUTH AFRICA 